\title{
A Potential Indication for Adjuvant Therapy in Node-Negative Esophageal Squamous Cell Carcinoma
}

\author{
Andrew M. Popoff, MD \\ Division of Thoracic Surgery, Department of Cardiothoracic Surgery, Henry Ford Hospital/Henry Ford Health System, \\ Detroit, MI
}

The National Comprehensive Cancer Network guidelines establish indications for adjuvant therapy in esophageal adenocarcinomas. ${ }^{1}$ The guidelines include consideration of high-risk features, such as lymphovascular invasion (LVI) and perineural invasion (PNI), among others. At present, guidelines for adjuvant therapy for resected squamous cell carcinomas of the esophagus are lacking due to clear evidence of therapeutic benefit. Guo and colleagues evaluate the potential significance of PNI and its possible importance on tailored adjuvant therapy for pNOM0 squamous cell carcinomas of the esophagus. ${ }^{2}$

The authors review a series of pNOMO resected esophageal squamous cell carcinomas and attempt to understand high-risk features that may represent indications for adjuvant therapy. They employ a relatively sophisticated methodology and subanalyses to conclude that Neural Invasion Index (NII) is the most consistent measure of PNI and that it affords greater fidelity than dichotomous PNI \pm . They further conclude that in this series LVI was not predictive of outcomes and that adjuvant radiotherapy in the high NII subset conferred a statistically significant survival advantage.

Perineural Invasion has previously been shown to negatively impact disease-free survival and disease-specific survival in esophageal squamous cell carcinomas. ${ }^{3}$ Gao et al. add to the body of evidence that tumor characteristics influence prognosis and could serve as targets for more

(C) Society of Surgical Oncology 2020

First Received: 7 June 2020

Accepted: 30 June 2020;

Published Online: 20 July 2020

A. M. Popoff, MD

e-mail: apopoff2@hfhs.org individualized adjuvant therapy. The work put forth addresses a specific subset of node-negative squamous cell carcinomas. While the authors use NII to better characterize perineural invasion and its potential impact on both prognosis and as an indication for adjuvant radiotherapy, it is not clear that this is applicable clinically. As the standard clinicopathologic evaluation at present for esophageal squamous cell carcinomas typically report perineural invasion in a dichotomous fashion. This may represent an opportunity for our colleagues in pathology to further refine their characterizations for ESCC.

The authors go on to report that in those with a high NII, and PNI, adjuvant radiotherapy can improve outcomes in those with pNOM0 ESCC. They posit that even patients with pT1-T2 tumors may benefit. Relatively few of the cohort received adjuvant chemotherapy, and they suggest this as a possible avenue of further exploration.

However, none of the T3-T4 subset received neoadjuvant chemoradiotherapy, and the authors acknowledge this. This comprises $81 \%(132 / 162)$ of the study population. It is likely that in current treatment paradigms, especially in the west, this subset would have received neoadjuvant therapy, and therefore the utility of adjuvant RT as a therapeutic possibility in this cohort is unclear. Those patient's having received neoadjuvant chemoradiation may not be candidates for further adjuvant radiotherapy.

Gao et al. provide a significant contribution to the body of literature covering esophageal squamous cell carcinoma. They present a possible opportunity to refine the adjuvant therapeutics based on high-risk features that may improve outcomes in a subset of patients. This work reinforces the subtleties and nuances of esophageal cancer care that thoracic surgeons and oncologists are working to understand. Perhaps more than anything else, it reminds us that there is still likely to be significant opportunity in this disease 
process to improve outcomes for what has traditionally been a pathology with dismal long-term survival. The authors create an opportunity for other research to confirm these results or explore adjuvant treatment for esophageal squamous cell carcinoma based on a better understanding of tumor characteristics and high-risk features that would include standard neoadjuvant therapy for T3-4 tumors in the study population.

\section{COMPLIANCE WITH ETHICAL STANDARDS}

CONFLICT OF INTEREST The authors declare that they have no conflict of interest.

\section{REFERENCES}

1. National Comprehensive Cancer Network (NCCN). NCCN clinical practice guidelines in oncology. Accessed June 2020.

2. Guo Y, Tian D, Gong Q, et al. Perineural invasion is a better prognostic indicator than lymphovascular invasion and a potential adjuvant therapy indicator in pNOM0 esophageal squamous cell carcinoma. Ann Surg Oncol. 2020. https://doi.org/10.1245/s10434020-08667-4.

3. Xu G, Feng F, Liu Z, et al. Prognosis and progression of ESCC patients with perineural invasion. Sci Rep. 2017;7:43828.

Publisher's Note Springer Nature remains neutral with regard to jurisdictional claims in published maps and institutional affiliations. 